\title{
Editorial:
}

\section{Diagnosis of viral diseases in India: at the cross roads}

In the last decade, epidemics of viral diseases have become one of the main concerns in public health. Apart from the ever threatening influenza and the relatively recent human immunodeficiency virus (HIV) infection, re-emergence of chikungunya fever and rapid increase in the incidence of dengue fever, emergence of new viral diseases like swine flu, have all contributed to this concern. The worrying mortality during epidemics and the morbidity due to post-infection sequelae along with lack of specific antiviral drugs or vaccines have generated a fear complex in the minds of common man and simultaneously threw challenges to the physicians and scientists in the areas of diagnosis and therapy of these viral infections.

Diagnosis is one of the prime requirements in disease management. Diagnosis of viral diseases is very crucial as it necessitates identification/estimation of either antigen or antigen specific antibodies produced by the immune system. Definitive diagnosis of viral diseases is essential for recognizing the occurrence of epidemics and for instituting appropriate treatment. Different types of diagnostic tests ranging from the basic level to the most advanced are currently available in the commercial market for the diagnosis of viral diseases. Broadly, diagnostic tests used for viral diseases can be classified in to: (i) tests involving direct specimen examination; (ii) viral isolation; and (iii) serodiagnostic tests.

In tests based on direct specimen examination, the clinical specimen is examined directly for the presence of virus antigen or viral genome using methods, such as, electron microscopy morphology/ immune electron microscopy, light microscopy histopathological appearances (e.g., presence of inclusion bodies), and molecular techniques for the detection of viral genomes. In tests based on virus isolation techniques, the infectious virus is isolated from the clinical sample and cultured in the embryonated eggs, animals or in cell-lines or cell-cultures. This is useful for further characterization of viruses or development of vaccine production. In serodiagnostic tests, several serological methods are being used to detect the viral antigens or their specific antibodies or other immunogens. In these serodiagnostic methods, diagnosis can be made by the detection of rising titres of antibody between acute and convalescent stage of infection or the detection of less specific immunoglobulin M (IgM) or immunoglobulin $\mathrm{G}$ (IgG). The various techniques used in serodiagnosis include complement fixation test (CFT), haemagglutination inhibition (HI) test, immunofluorescence (IF) techniques, neutralization tests, single radial haemolysis, enzyme linked immunosorbent assay (ELISA), particle agglutination, western blot, recombinant immunoblot assay (RIBA) and line immunoassay. Expertise is essential to use the above techniques and these have become part of many advanced clinical virology laboratories.

In general, the majority of common viral infections (e.g., viral hepatitis and HIV) can be diagnosed in clinical specimens by using serodiagnostic methods. However, in recent times, these traditional diagnostic techniques are slowly becoming less useful or relevant in the management due to the nonspecificity in detecting various strains/ pathotypes or isolates or serotypes of the viruses. This is especially true for the recently emerging viral diseases like swine flu, Nipah virus, Chikungunya virus etc., to name a few. This is paving the way for more interest in molecular methods for the detection of viral genome and these are getting established as the future diagnostic tests in virology. The viral load and antiviral resistance or subtyping assays are now part of the biological monitoring of patients chronically infected by several viruses. But viral genome studies are not yet used in routine diagnostic work in a clinical virology laboratory. In the $21^{\text {st }}$ century the molecular biology techniques for the detection of viral genomes like the nucleic probe based or genome amplification based methods e.g., polymerse chain reaction (PCR) are expected to play an increasingly important role in the clinical virology laboratory. PCR is the only amplification technique which is in common 
use and an extremely sensitive technique. It is possible to achieve a sensitivity to detect a single DNA molecule in a clinical specimen. PCR is being used increasingly for viral diagnosis in super specialty clinics and there is availability of closed automated systems that could also perform quantification e.g., real-time PCR (RT-PCR). This is expected to solve major part of diagnostic confusion that is now prevailing in the identification of causative virus.

Classical molecular techniques such as dot-blot and Southern-blot depend on the use of specific DNA/RNA probes for hybridization. The specificity of the reaction depends on the conditions used for hybridization. These techniques may allow for the quantification of DNA/RNA present in the specimen. However, it is often found that the sensitivity of these techniques is not better than conventional viral diagnostic methods. Recent molecular techniques such as the polymerase chain reaction (PCR), ligase chain reaction (LCR), nucleic acid based amplification (NASBA), loop mediated isothermal amplification (LAMP) and branched DNA (bDNA) depend on some form of amplification, either the target nucleic acid, or the signal itself. bDNA is essentially a conventional hybridization technique with increased sensitivity. Other amplification techniques such as LCR and NASBA are also being used to detect the viruses. Recently the microarray technique is being used for detection of viruses in large scale.

Due to the viral genome variability or diversity in the environment, several strains of a virus emerge. Due to this, the diagnostic utility of commercially available viral diagnostic tests is often seriously impaired. This becomes the crux of the problem for diagnosing viral infection precisely even with advanced techniques. Another drawback in the application of viral diagnostics in a clinical setting is that the patient may come to the hospital during window period wherein absence of viral specific antibodies results in a negative test report adding to the clinician's confusion. In Indian scenario, lack of awareness on viral diagnostic methods and limited access to viral diagnostic laboratory could also contribute to underutilization of viral diagnostic tests by clinicians. Lack of expertise, nonavailability of needed equipment and excessive cost are additional factors of concern. There is a need to address these issues and the measures should include enhancing the awareness among clinicians regarding the various diagnostic methods and developing algorithms for choosing the most appropriate test(s) for ascertaining the virological diagnosis.

Keeping in view the recent outbreaks of several viral diseases in India, the Government of India has started concentrating more on the viral infections, especially the vector borne diseases by launching national vector borne disease control program. At present, the main thrust is on control of vectors. It is expected that further growth of these program would obviously focus on better diagnosis in a manner similar to National AIDS Control Program. We can see this in the twelfth plan. ${ }^{1}$ However, it would also be necessary to focus more in training clinicians and popularizing the use of appropriate diagnostic methods for early diagnosis of the viral diseases. There is also a need to develop molecular diagnostic kits in India so as to contain costs.

\section{D.V.R. Sai Gopal \\ Professor and Head Department of Virology \\ Sri Venkateswara University \\ Tirupati-517502, India \\ email: dvrsaigopal@gmail.com}

Received: 12 September,2012.

Sai Gopal DVR. Diagnosis of viral diseases in India: at the cross roads. J Clin Sci Res 2012;1:155-6.

\section{REFERENCES}

1. Report of working group on disease burden for the twelfth five year plan - wg-3 (1) - communicable diseases dated 30th July 2011. Available at URL: http//planningcommission.nic.in/aboutus/committee/wrkgrp12/health/ WG_3_1communicable.pdf.Accessed on September 11, 2012. 